\title{
Transfusion Reaction Reporting Culture in Hemovigilance Program of India since Its Inception
}

\author{
${ }^{1}$ Akanksha Bisht, ${ }^{2}$ Shakti Kumar Gupta, ${ }^{3}$ Surinder Singh
}

\begin{abstract}
Hemovigilance Program of India (HvPI) at the national level was launched on 10th December 2012 by National Institute of Biologicals (NIB), Noida, Ministry of Health and Family Welfare, Government of India as the National Coordinating Centre (NCC). Awareness about the program, its objectives and its nonpunitive implications are being generated through organizing continuing medical educations (CMEs) on HvPI in different regions of the country from time to time. A total of 24 CMEs on HvPI have been organized all across the country. The study shows that creating awareness about this progam among healthcare professionals has resulted in substantial increase in number of center enrolled under HvPI as well as number of reporting of transfusion reactions under HvPI since the inception of this program.
\end{abstract}

Keywords: Clinicians and nurses, Continuing medical education, Hemovigilance, Reporting culture, Transfusion reactions.

How to cite this article: Bisht A, Gupta SK, Singh S. Transfusion Reaction Reporting Culture in Hemovigilance Program of India since Its Inception. Int J Res Foundation Hosp Healthc Adm 2015;3(2):69-70.

Source of support: Nil

Conflict of interest: None

\section{INTRODUCTION}

Although blood transfusions can be life-saving, they are not without risks. Blood is being tested using advance technologies which has resulted in decrease risk of transfusion transmitted viral disease. However, there are variety of transfusion risks involved which cannot be entirely eliminated, several of which are noninfectious in nature. ${ }^{1-4}$

\footnotetext{
${ }^{1}$ Officer-in-Charge, ${ }^{2}$ Medical Superintendent, ${ }^{3}$ Director

1,3 Hemovigilance Program of India Cell, National Institute of Biologicals, Ministry of Health and Family Welfare, Government of India, Noida, Uttar Pradesh, India

${ }^{2}$ Department of Hospital Administration, All India Institute of Medical Sciences, New Delhi, India
}

Corresponding Author: Akanksha Bisht, Officer-in-Charge Hemovigilance Program of India Cell, National Institute of Biologicals, Ministry of Health and Family Welfare, Government of India, A-32, Sector-62, Noida-201309, Uttar Pradesh, India e-mail: akankshabisht83@gmail.com
Hemovigilance is an important tool for improving blood safety. Hemovigilance data provides valuable information wrt transfusion reactions in recipient of blood transfusion as well as complications in donors. It is defined as a set of surveillance procedures covering the whole blood transfusion chain from the collection of the blood and its components to the follow-up of its recipients, intended to collect and access information on unexpected or undesirable effects resulting from the therapeutic use of labile blood products, and to prevent its occurrence and reoccurrence. ${ }^{5}$ A centralized hemovigilance program to assure patient safety and to promote public health was launched for the first time in India on December 10, 2012, in 60 medical colleges in the first phase along with a wellstructured program for monitoring adverse reactions associated with blood transfusion and blood product administration. National Institute of Biologicals (NIB) is the National Coordinating Center (NCC) for Hemovigilance Program of India (HvPI). ${ }^{6}$

\section{MATERIALS AND METHODS}

A total of 2737 transfusion reactions have been reported to HvPI since the inception of this program, i.e. from 10th December 2012 till 31st August 2015 and were analyzed. These reports are being forwarded through a hemovigil software which facilitate collection and collation of data in transfusion reaction reporting form (TRRF) from various centers across the country and transmit this data to NCC at NIB? ${ }^{7}$

A total of 24 continuing medical educations (CMEs) on HvPI have been organized all across the country. During the year 2012 to 2013, CMEs on hemovigilance were organized mainly for the personnel involved in blood banks and transfusion medicines departments. However, it was felt that, since the clinicians and nurses are important link to success of this program, dissemination information about the importance and need of the program may boost up the reporting to HvPI. The first CME on hemovigilance for clinicians was organized on 26th April 2014 at Government Medical College, Chandigarh. Thereafter, in every CME on hemovigilance organized by NIB, clinicians and nurses were actively involved as participants, panelists and speakers. 


\section{OBSERVATIONS}

On analyzing the transfusion reaction, data reported to HvPI, it was observed that, out of 226 centers enrolled under hemovigilance program, 76 centers are actively reporting. The reporting centers included blood banks of medical institute (government/private), hospitals (government/private/charitable) and standalone blood banks. A total of 31 centers out of 76 centers reporting centers were from government medical colleges/hospitals, i.e. $40.78 \%$, and rest 45 centers were from private/ charitable/standalone blood banks, i.e. 59.22\% (Fig. 1).

This study revealed that the number of centers enrolled during 1st year after the inception of the program were 117 (2012-2013), which increased to 184 for the second year (2013-2014) and till date 226 centers are enrolled under this program. Also number of transfusion reactions reported to hemovigilance program of India were 477 during 1st year after the inception of the program, which increased to 1665 for the second year (2013-2014) and till date 2737 transfusion reactions have been reported to this program.

It was observed that there was an increase in number of centers being enrolled under HvPI and the rate of reporting were also consistently increasing after dissemination information through HvPI newsletter, pamphlets, awareness programs and circulating updates to the center via e-mails (Graph 1). Also, involving clinicians and nurses in the awareness programs resulted in more

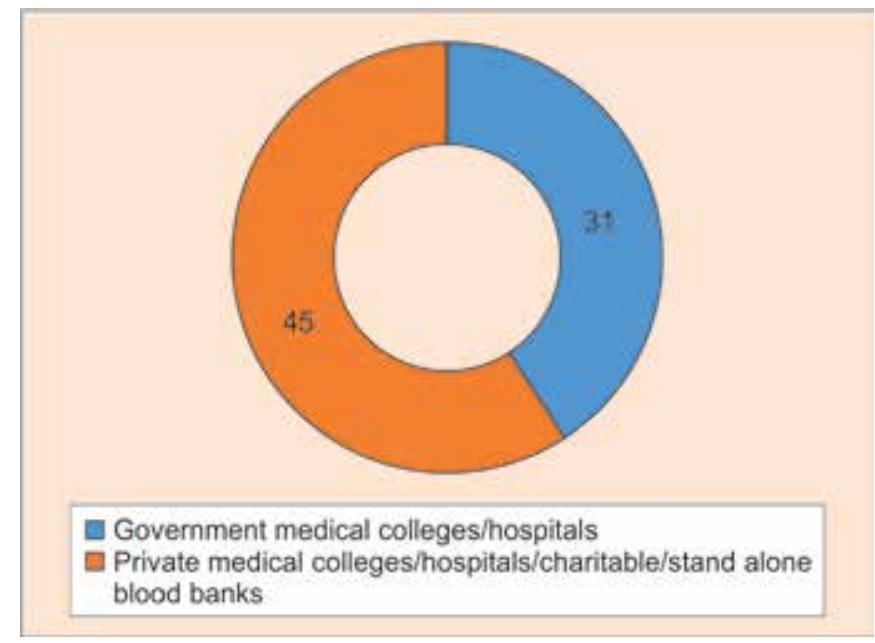

Fig. 1: Number of reporting centers

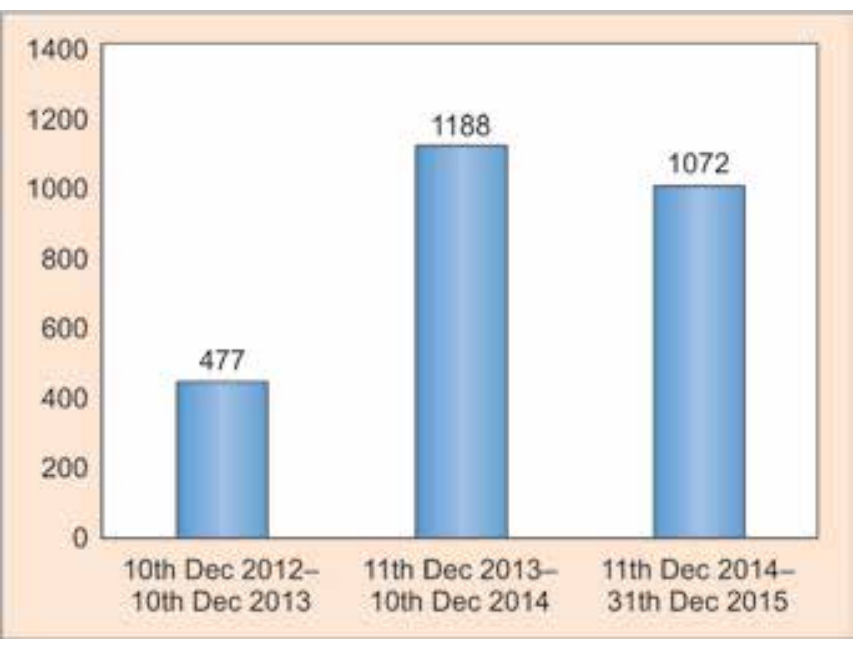

Graph 1: Number of transfusion reaction reported under HvPI

number of centers being enrolled, and hence increased the frequency of reporting of transfusion reactions.

\section{CONCLUSION}

This study may be useful in devising strategies to create awareness about the program among healthcare professional and devising format to assess the problems wrt reporting of transfusion reaction by the enrolled centers under the hemovigilance program. Further, when compared the progress of this programs since its inception, i.e. from 10th December 2015, it is evident that awareness programs to sensitize the healthcare professionals is an essential tool to improve and strengthen the hemovigilance.

\section{REFERENCES}

1. Busch MP. HIV, HBV and HCV: new developments related to transfusion safety. Vox Sang 2000;78(2):253-256.

2. Dodd RY. Current viral risks of blood and blood products. Ann Med 2000;32(7):469-474.

3. Perrotta PL, Snyder EL. Non-infectious complications of transfusion therapy. Blood Rev 2001;15(2):69-83.

4. Eder AF, Chambers LA. Non-infectious complications of transfusion. Arch Pathol Lab Med 2007;131:708-718.

5. International Haemovigilance Network. Available at: http:// www.ihn-org.com/.

6. Bisht A, Singh S, Marwaha N. Hemovigilance program-India. Asian J Transfus Sci 2013;7(1): 73-74.

7. Marwaha N, Bisht A, Singh S. Setting up haemovigilance from the very first step: the Indian perspective. ISBT Science Series 2014;9(1):178-183. 